\title{
PARTIAL TRISOMY FOR 19q DUE TO PATERNAL 17/19 RECIPROCAL TRANSLOCATION
}

\author{
Harumichi Madokoro, Shozo OHdo, Tohru Sonoda, \\ Kenji Kawaguchi, and Ken-ichi OHBa \\ Department of Pediatircs, Miyazaki Medical College, \\ Miyazaki 889-16, Japan
}

\begin{abstract}
Summary A malformed female infant having karyotype 46,XX,der(17), $\mathrm{t}(17 ; 19)(\mathrm{q} 25.3 ; \mathrm{q} 13.3)$ pat is reported. She had following abnormalities: low birth weight, congenital heart disease, microcephaly, high frontal hairline, short nose with flat nasal root, cleft lip and palate, broad mouth with downturned commissures, short neck with excess skin, clinodactyly of 5th fingers, sacral dimple, wide set nipples, and unilateral palmar transverse crease. Autopsy revealed congenital heart disease and polysplenia, but no abnormality was found in other organs. Her sister was stillborn, with cleft lip and palate. Our case suggests that fatality due to severely malformed internal organs may occur in duplication at the distal third of $19 \mathrm{q}$.
\end{abstract}

\section{INTRODUCTION}

For the diagnosis of a chromosome aberration, especially of an autosomal aberration, mental retardation as well as the pattern of abnormality is informative (Schinzel, 1984). Cases of 19q duplication are rare, and since all cases have resulted from parental translocation, the kind and frequency of complicated abnormalities is not yet known.

\section{CASE REPORT}

The proband, a female infant, was born on March 19, 1987 as a second child to healthy and unrelated parents. When she was born, the father was 31 , and the mother, 28. Her sister was stillborn, at a birth weight of $1,610 \mathrm{~g}$. She had cleft lip and palate. Chromosome analysis and autopsy were not carried out.

The birth weight of the proband was $2,350 \mathrm{~g}$, height $41.0 \mathrm{~cm}$, and head circumference $29.0 \mathrm{~cm}$. She was referred to our outpatient clinic on March 27, 1987 for multiple congenital abnormalities and cyanosis. Her weight at admission was 


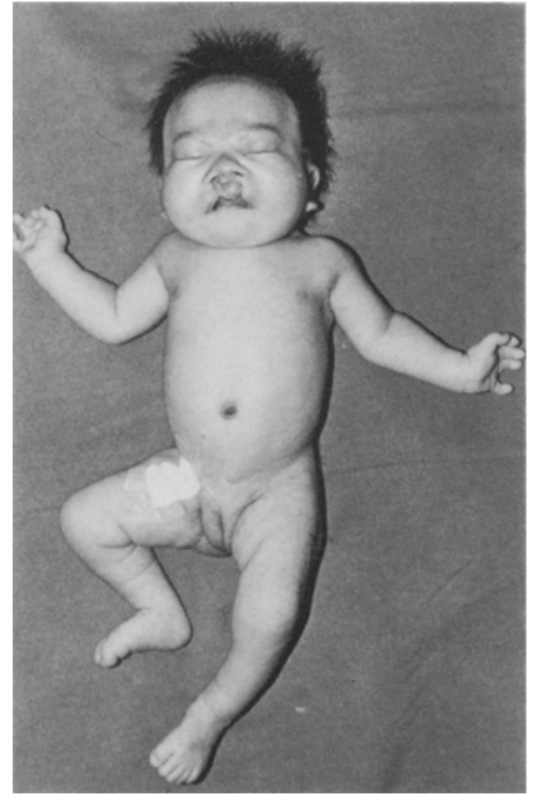

Fig. 1. Proband, at the age of 7 days.

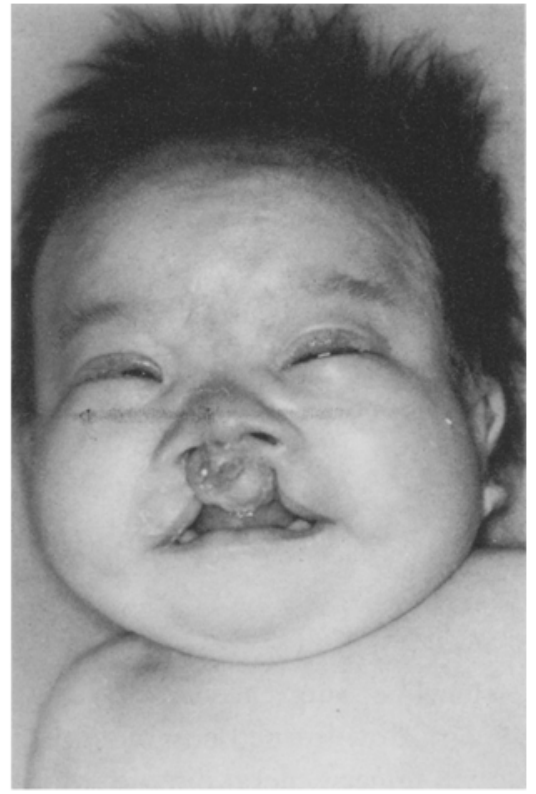

Fig. 2. Facial appearance of the proband.

$2,276 \mathrm{~g}(-2.2 \mathrm{SD})$, height $42.0 \mathrm{~cm}(-4.1 \mathrm{SD})$, and head circumference $29.5 \mathrm{~cm}$ ( -2.3 SD). Physical examination revealed the following abnormalities (Figs. 1 and 2): high frontal hairline, blepharoptosis, short nose with flat nasal root, bilateral cleft lip and palate, broad mouth with downturned commissures, short neck with excess skin, sacral dimple, clinodactyly of the 5th fingers and wide set nipples.

A grade $2 / 6$ continuous murmur was heard at the upper left sternal border. Dermatoglyphic study showed finger patterns consisting of eight ulnar loops, one whorl and one arch. On the palms, right palmar transverse crease was observed, but bilateral atd angles were within normal range $\left(40^{\circ}\right.$ on both palms). Echocardiography revealed single ventricle and patent ductus arteriosus.

Although treatment for both heart failure and feeding difficulty was continued after admission, the patient died on the 71st day after birth.

Autopsy revealed congenital heart defects consisting of single ventricle, patent ductus arteriosus, double outlet right ventricle, pulmonary atresia, atrial septal defect, single coronary artery, persistant fetal circulation and persistent superior vena cava. There was no abnormal lobation and branching in the lung, and the diaphragm was normal. In the abdomen, mesenterium commune and polysplenia were noted. No abnormalities were observed in the liver, gallbladder and ovary.

Leukocyte cultures from peripheral blood showed an extra band on the terminal region of the long arm of chromosome 17 (Fig. 3). While the karyotype of the mother was normal, that of the father was $46, X Y, r c p(17 ; 19)$ (q25.3;q13.3) (Fig. 4). 


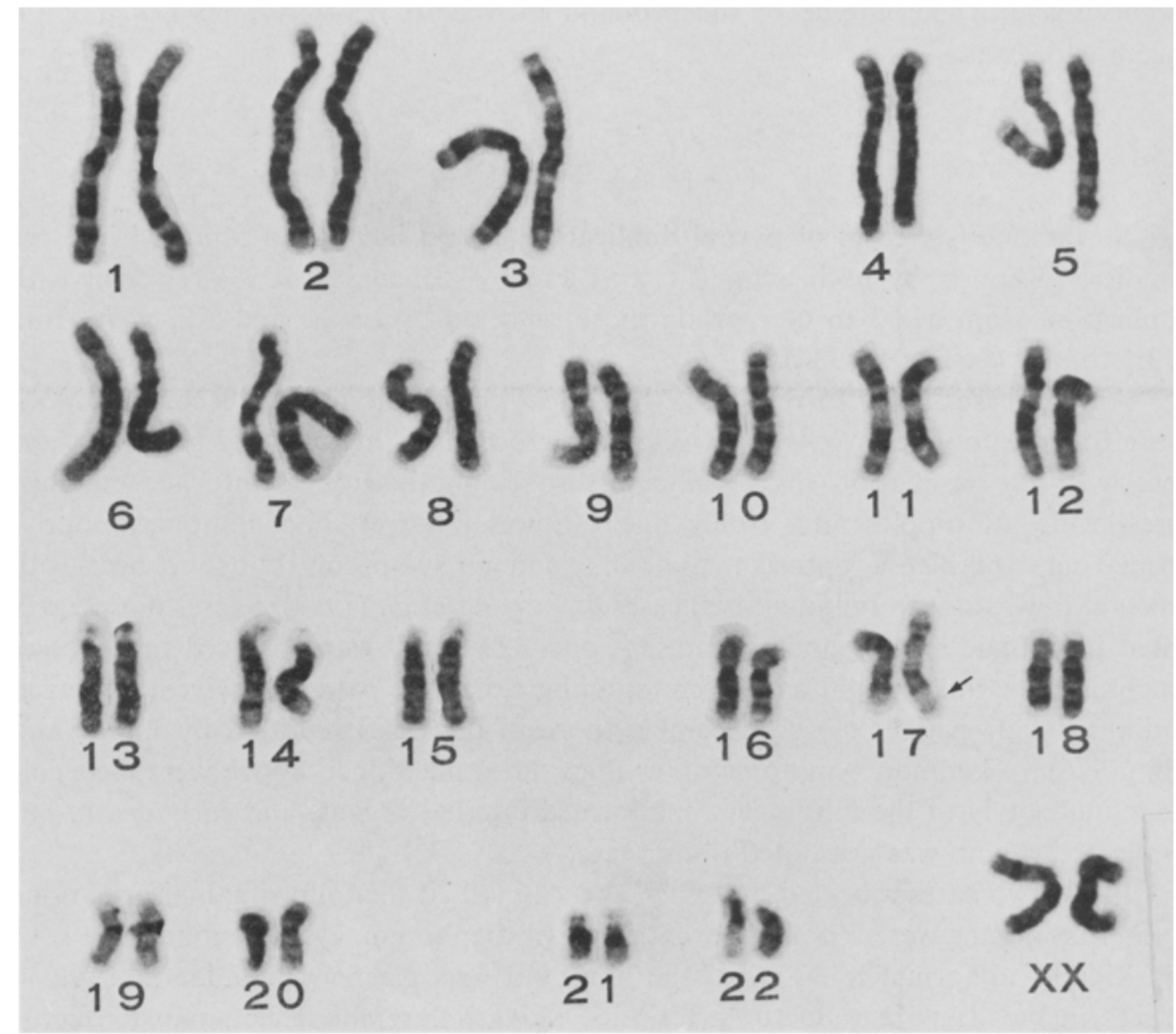

Fig. 3. Karyotype of the proband. $46, X \times, 17 q+$.

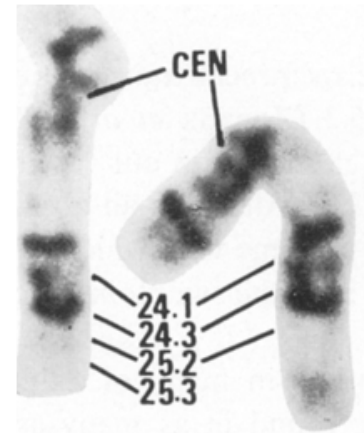

17

$\operatorname{der}(17)$

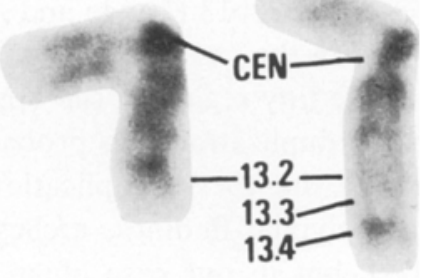

$\operatorname{der}(19)$

Fig. 4. Partial karyotype of the father (high resolution G-banded). 46,XY,rcp $(17 ; 19)$ (q25.3;q13.3). 
Accordingly, the karyotype of the proband proved to be $46, X X, \operatorname{der}(17), t(17 ; 19)$ (q25.3; 1 13.3)pat.

\section{DISCUSSION}

So far only six cases of partial duplication at $19 \mathrm{q}$ have been reported in three families. Reports by both Schmid (1979) and by Zonana et al. (1987) dealt with duplication from q13.3 to qter, while those reported by Lange and Alfi were from q13.1 to qter (Schinzel, 1984).

Among cases hitherto reported, the following symptoms are in common with those found in our case: low birth weight, microcephaly, high frontal hairline, short nose with flat nasal root, short philtrum, broad mouth with downturned commissures, wide set nipples and widely open sutures. Barrel shaped thorax, poorlyformed ears and blepharoptosis may be the common symptoms, but short neck with excess skin was not found in all two cases of Zonana et al. (1982). Sacral dimple was noted in Schmid's cases and the present one. Epilepsy was observed in all cases except in ours, but it might have been found here, had the patient survived. Clinical findings in our patient closely resemble those in the cases reported by Lange and Alfi (1976). Common symptoms other than those mentioned above were cleft palate, clinodactyly of the 5th fingers, malformed internal organs, and early death, but duplicated thumb was not noted in our case.

In the two cases reported by Lange and Alfi (1976) the following malformations in internal organs were reported: eventration of diaphragm, cystic lesion of the liver and kidney, abnormal lobation of the lung and hypoplastic gallbladder. Cardiac defects included: patent ductus arteriosus and atrial septal defect, patent ductus arteriosus and valvular pulmonic stenosis. In comparison with our case, all of these were mild. In our case mesenterium commune and polysplenia were noted, but other organs were normal. Congenital heart disease, albeit of different severity, was the only malformation of internal organs in common with our case and those of Lange and Alfi (1976).

The other chromosomal sites involving in the reciprocal translocation together with $19 \mathrm{q}$ in the parents of probands were 17q25.3 (Zonana et al., 1982), 20pter (Schmid, 1979), and 22p13 (Lange and Alfi, 1976). The site in our case was exactly the same as that in the cases of Zonana et al. (1982). Although all observers noted monosomy of the tiny region in the opposite chromosome with which translocation was made in $19 \mathrm{q}$ duplication, it is probable that at least the symptoms in common to all cases might be due to $19 \mathrm{q}$ duplication.

In dermatoglyphic findings, arches were counted in many of Schmid's cases (Schmid, 1979), but in our case ulnar loops were found in as many as 8 fingers. Right palmar transverse crease was noted in our case, but it was not described in the other cases.

Since there have been few reported cases of $19 q$ duplication, the kinds and fre- 
quencies of complicated abnormalities are as yet not clear. In the duplication from $19 \mathrm{q} 13.3$ to qter in our case, it was suggested that early death was due to severely malformed internal organs.

\section{REFERENCES}

Lange, M. and Alf, O.S. 1976. Trisomy 19q. Ann. Genet. 19: 17-21.

Schinzel, A. 1984. Catalogue of Unbalanced Chromosome Aberrations in Man, Walter de Gruyter, New York, pp. 669-671.

Schmid, W. 1979. Trisomy for the distal third of the long arm of chromosome 19 in brother and sister. Hum. Genet. 46: 263-270.

Zonana, J., Brown, M.G., and Magenis, R.E. 1982. Distal 19q duplication. Hum. Genet. 60: $267-270$.

Vol. 33, No. 1, 1988 